\title{
Relapse of ulcerative proctocolitis during treatment with non-steroidal anti-inflammatory drugs
}

\author{
D. S. RAMPTON* \\ D.Phil., M.R.C.P. \\ G. E. Sladent \\ D.M., F.R.C.P. \\ *University College London Medical School, Rayne Institute, University Street, London WC1E 6JJ, and \\ †Guy's Hospital, London SEI 9RT
}

\begin{abstract}
Summary
In four patients with inactive ulcerative proctocolitis, oral administration of non-steroidal anti-inflammatory drugs appeared to precipitate relapse. These observations suggest that inhibitors of prostaglandin synthesis should be used with care in patients with this disease, and are consistent with the hypothesis that prostaglandins may have a protective role in the lower as well as the upper gastrointestinal tract.
\end{abstract}

\section{Introduction}

Rectal mucosal prostaglandin synthesis is increased in active ulcerative colitis (Sharon et al., 1978; Harris, Smith and Swan, 1978). The possibility that this change could be of pathogenetic importance has led to the proposal that patients with active disease might benefit from treatment with those of the non-steroidal anti-inflammatory agents which inhibit prostaglandin synthesis. In this report, however, 4 patients with quiescent proctocolitis are described in whom oral administration of such drugs for painful disorders appeared to precipitate relapse.

\section{Case histories}

These are outlined in Table 1. In each patient, relapse, characterized by increased stool frequency, rectal bleeding and a deterioration in mucosal appearance at sigmoidoscopy, occurred soon after the oral administration of a non-steroidal antiinflammatory agent; remission was re-established within 4 weeks of stopping the drug, in 2 cases ( 3 and 4) after the introduction of conventional treatment. Although no patient was re-challenged with the same drug, brief reversible relapses did occur in Case 1 after subsequent sequential atempts at oral treatment of his ankylosing spondylitis with diclofenac, flurbiprofen and naproxen.

\section{Discussion}

Non-steroidal anti-inflammatory drugs predispose to ulceration in the upper gastrointestinal tract.
Because this effect can be prevented, at least in experimental animals, by pretreatment with prostaglandins, it has been suggested that such ulceration is related to drug-induced mucosal prostaglandin deficiency, and that these ubiquitous fatty acids may play a cytoprotective role in the stomach and small intestine (Robert, 1979).

However, although the manufacturers of indomethacin advise special care in its prescription to patients with inflammatory bowel disease (ABPI Data Sheet Compendium, 1980-81), there is very little published information on the effect of inhibitors of prostaglandin synthesis on the large intestine. Thus, while Levy and Gaspar (1975) described a single patient in whom indomethacin suppositories produced proctitis, and Coutrot et al. (1978) reported 2 patients in whom perforation of colonic diverticula occurred in association with the oral ingestion of indomethacin, the authors are unaware of any previous published reports of symptomatic and sigmoidoscopic deterioration in patients with inactive ulcerative colitis following the oral administration of non-steroidal anti-inflammatory agents.

The apparently detrimental effect of prostaglandin synthesis inhibitors in these patients with quiescent disease resembles that found in a short-term therapeutic trial of flurbiprofen in patients with active proctocolitis (Rampton and Sladen, 1981), and fails to support the proposal that such drugs might prove beneficial in ulcerative colitis (Kimberg, 1978; Sharon et al., 1978). Indeed, these observations suggest that, as in the upper gastrointestinal tract, prostaglandins could be cytoprotective in the large bowel, a function which would be consistent with the theory that sulphasalazine maintains remission in quiescent colitis by inhibiting degradation, rather than synthesis, of mucosal prostaglandins (Hoult and Moore, 1978).

As well as impairing mucosal cytoprotection, prostaglandin synthesis inhibitors could increase rectal bleeding by interfering with platelet function 


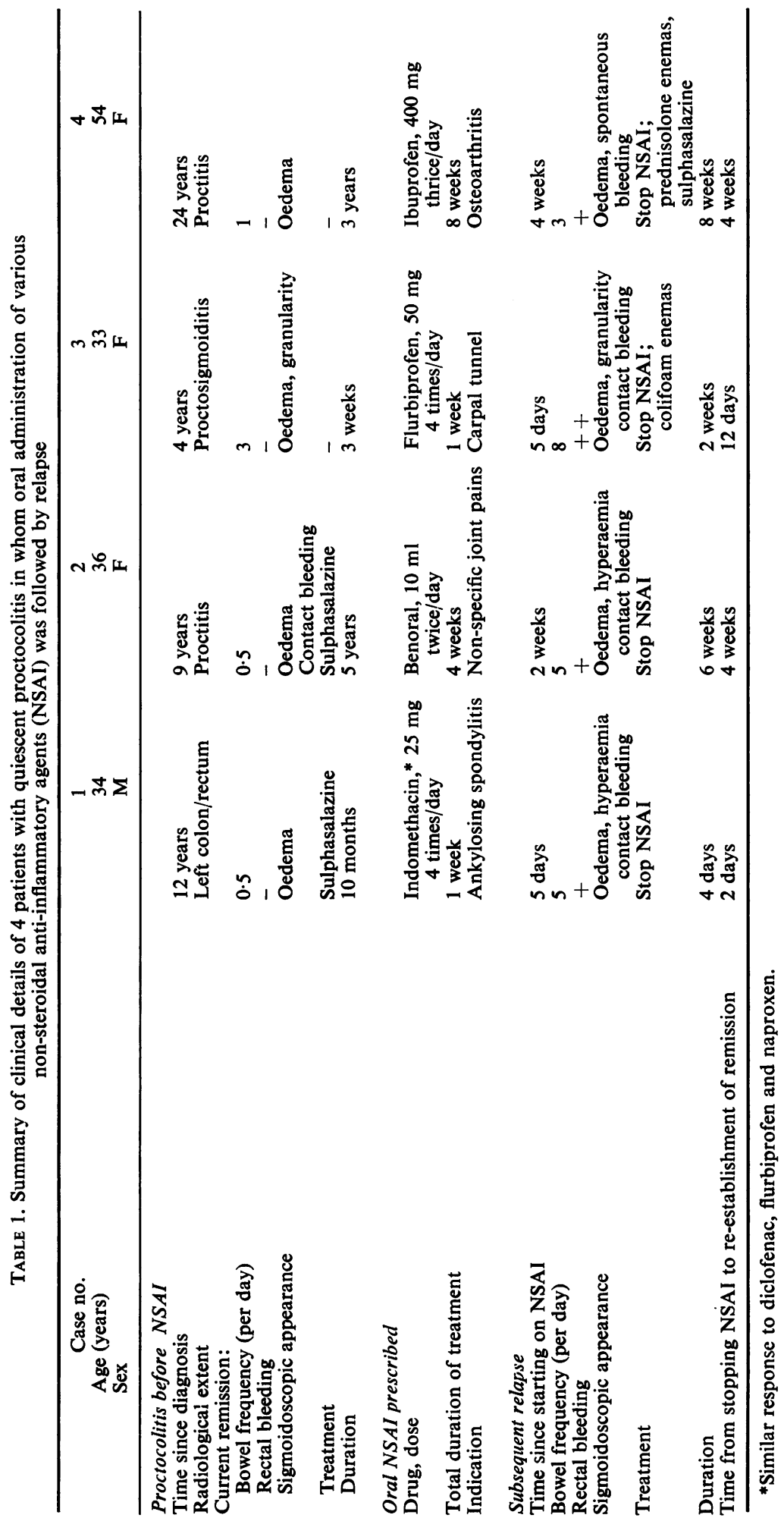


(Cockbill, Heptinstall and Taylor, 1979); more speculatively, they could also lead to mucosal damage by enhancing synthesis of products of the lipoxygenase enzyme system (Moncada and Vane, 1979).

Non-steroidal anti-inflammatory agents are frequently used in the treatment of intercurrent or associated painful conditions in patients with ulcerative colitis. Whether or not the effects described here are related to alterations in prostaglandin metabolism, it would seem appropriate that prescribers bear in mind the possibility that oral as well as local administration of this group of drugs may induce or exacerbate relapse in some patients with this disease.

\section{References}

ABPI (1980-81) Data Sheet Compendium, p. 717. Datapharm Publications, London.

Cockbill, S.R., Heptinstall, S. \& Taylor, P.M. (1979) A comparison of the abilities of acetylsalicylic acid, flurbiprofen and indomethacin to inhibit the release reaction and prostacyclin synthesis in human blood platelets. British Journal of Pharmacology, 67, 73.
Coutrot, S., Roland, D., Barbier, J., van der MarcQ, P. Alcalay, M. \& Matuchansky, C. (1978) Acute perforation of colonic diverticula associated with short-term indomethacin. Lancet, ii, 1055.

Harris, D.W., Smith, P.R. \& Swan, C.H.J. (1978) Determination of prostaglandin synthetase activity in rectal biopsy material and its significance in colonic disease. Gut, 19, 875.

HoulT, J.R.S. \& Moore, P.K. (1978) Sulphasalazine is a potent inhibitor of prostaglandin 15-hydroxydehydrogenase: possible basis for therapeutic action in ulcerative colitis. British Journal of Pharmacology, 64, 6.

KimBERG, D.V. (1978) The ubiquitous prostaglandins and their role in ulcerative colitis. Gastroenterology, 75, 748.

LEVY, N. \& GASPAR, E. (1975) Rectal bleeding and indomethacin suppositories. Lancet, i, 577.

MonCADA, S.H. \& VANE, J.R. (1979) Mode of action of aspirin-like drugs. Advances in Internal Medicine, 24, 1.

Rampton, D.S. \& Sladen, G.E. (1981) Prostaglandin synthesis inhibitors in active ulcerative colitis: flurbiprofen compared with conventional treatment. Prostaglandins (in press).

ROBERT, A. (1979) Cytoprotection by prostaglandins. Gastroenterology, 77, 761 .

Sharon, P., Ligumsky, M., Rachmilewitz, D. \& Zor, U. (1978) Role of prostaglandins in ulcerative colitis. Enhanced production during active disease and inhibition by sulphasalazine. Gastroenterology, 75, 638. 